\title{
True Single Phase Clocking Flip-Flop Design using Multi Threshold CMOS Technique
}

\author{
Priyanka Sharma \\ ME (ECE) Student \\ NITTTR Chandigarh
}

\author{
Rajesh Mehra \\ Associate Professor \\ Department of ECE \\ NITTTR Chandigarh
}

\begin{abstract}
This paper enumerates the design of low power and high speed double edge triggered True Single Phase Clocking (TSPC) D- flip-flop. The TSPC CMOS flip-flop uses only one clock signal that is never inverted and it eliminates the clock skew. The originally developed TSPC flip-flop are very sensitive to the clock slope and large portion of power is spent in pre-charging the internal nodes, which makes TSPC dynamic circuits less power efficient. In the conventional CMOS design, high leakage current is becoming a significant contributor to power dissipation. To overcome the existing problem of CMOS TSPC D flip-flop, a Multi-threshold CMOS (MTCMOS) technology is used for leakage minimization. The designed flip-flops are compared in terms of power consumption and propagation delay and power delay product and simulations are carried out by MICROWIND 3.1 tools. The proposed MTCMOS designs such as original MTCMOS implmentation and NMOS insertion in MTCMOS design of TSPC D flip-flop saves static power $57.517 \%$ and $58.871 \%$ as compared to conventional DE-TSPC D flip-flop respectively at $1.2 \mathrm{~V}$.
\end{abstract}

\section{Keywords}

DE-TSPC flip-flop, MTCMOS, power dissipation, figure of merit (FOM), BSIM.

\section{INTRODUCTION}

Flip- flop are the fundamental building block of the digital electronics systems used in computers, microprocessors and many other types of systems. Flip-flop can be either simple or clocked; data is stored in flip-flop at each rising and falling edge of clock signal so that it can be applied as inputs to other combinational or sequential circuits, such flip-flops that store data on rising or falling edge of clock are referred as single edge triggered flip flops and the flip-flops that store data on both the rising and falling edge of a clock pulse are referred as double edge triggered flip-flops. Among all the commonly used storage elements, the timing behaviors of edge-triggered flip-flops (ETFFs) are the simplest [1]. In the earlier period power consumption was a peripheral consideration for designers; the VLSI designers were more bent towards the performance and area of the circuits. In recent years, however, this has begun to change rapidly and power is being given equal importance in comparison to area and speed [2]. There is always a trade- off between power and performance [3]. In CMOS circuit there are 3 sources of power dissipation, static (leakage) power dissipation, short circuit power and dynamic power dissipation [4]. There are two fully dynamic flip-flops- one is TSPC flip-flop and another is dynamic transmission gate flip- flops. True single phase clocking D flip-flop has several advantages as it eliminates the clock skew caused by different clock phases and clock signals are generated off-chip which significantly saves chip area and power consumption. But large portion of power is spent in pre-charging the internal nodes, which makes TSPC circuits less power efficient. These fast flip-flops are more sensitive to power supply noise and leakage current [5].

The sole source of power consumption in CMOS circuit is the leakage current. In the conventional CMOS flip-flop design, high leakage current is becoming a significant contributor to power dissipation and this result in the reduction of threshold voltage, channel length and gate oxide thickness [6]. The identification of different leakage components and their modeling is very important for the design of low power and high speed applications. Leakage is related to the current that flows when gate terminal is not changing. The current that flows from power supply to ground nodes is known as static biasing current. And static current that flows from power supply to ground nodes without any degradation in input is known as leakage power and it is given as$P_{\text {stat }}=I_{\text {stat }} \cdot V_{D D}$

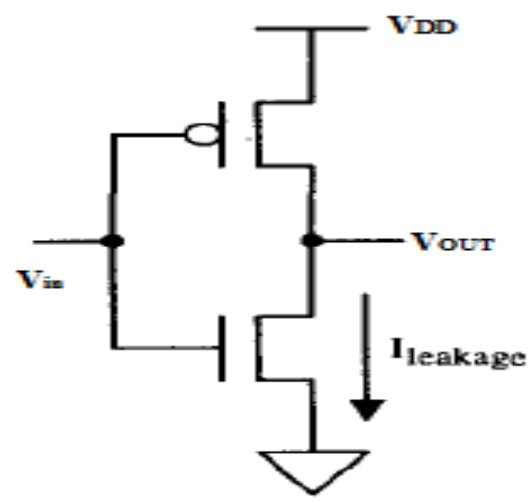

Fig. 1 Leakage Current in the CMOS Inverter

MTCMOS is the variation of CMOS chip technology which has the transistors with multiple threshold voltage in order to optimize delay or power. Multiple threshold CMOS technique can be used for reducing leakage currents in low -voltage devices in the stand by modes this is done by using both PMOS and NMOS with two different threshold voltages in the circuitry. Multiple threshold CMOS technique use low threshold voltage $\left(\mathrm{V}_{\mathrm{t}}\right)$ transistors for computation and a high threshold voltage $\left(\mathrm{V}_{\mathrm{t}}\right)$ transistors as a switch to disconnect the power supply during idle mode.

This paper is organized as follows: Section 2 discusses design of true single phase clocking D flip-flop. Section 3 presents working principle of multi threshold CMOS (MTCMOS). Section 4 presents the proposed schematic design of DETSPC D flip-flop is based on MTCMOS technique. Section 5 presents layout simulation in which the layout design rule describes how the small features can be and how closely they 
are packed into a chip. Section 6 describes results \& comparison this will present an extensive comparison of different designs of dynamic TSPC D flip-flops. Section 7 concludes the paper and presents the future directions.

\section{TRUE SINGLE PHASE CLOCKING D FLIP-FLOP}

The true single-phase clock (TSPC) is a common dynamic flip-flop variety which performs the flip-flop operation at high speeds and with little power. TSPC latch developed by Yuan and Svensson (Yuan and Svensson 1989) is a fast and simple structure that uses a single-phase clock. This latch was constructed by merging two parts consisting of CMOS Domino and CMOS NORA logic (Goncalves and De Man 1983). Previously double edge triggered flip-flop were designed using two complementary latches in parallel. One latch was reacting on positive edge of square clock pulse and the other was reacting at the negative edge. Then the outputs of two latches were combined into a single output using a merging circuit. Within the merging circuit, when the output of one of the circuit is high impedance node, it lets the other circuit decide the output value and vice versa.

Double edge triggered TSPC flip-flop was introduced by Wang [9] which uses only eighteen transistors. This conventional design of double edge triggered TSPC flip-flop is shown in figure 2. But in this design an extra CMOS inverter is added at the output to obtain a true logic and not the complemented logic.

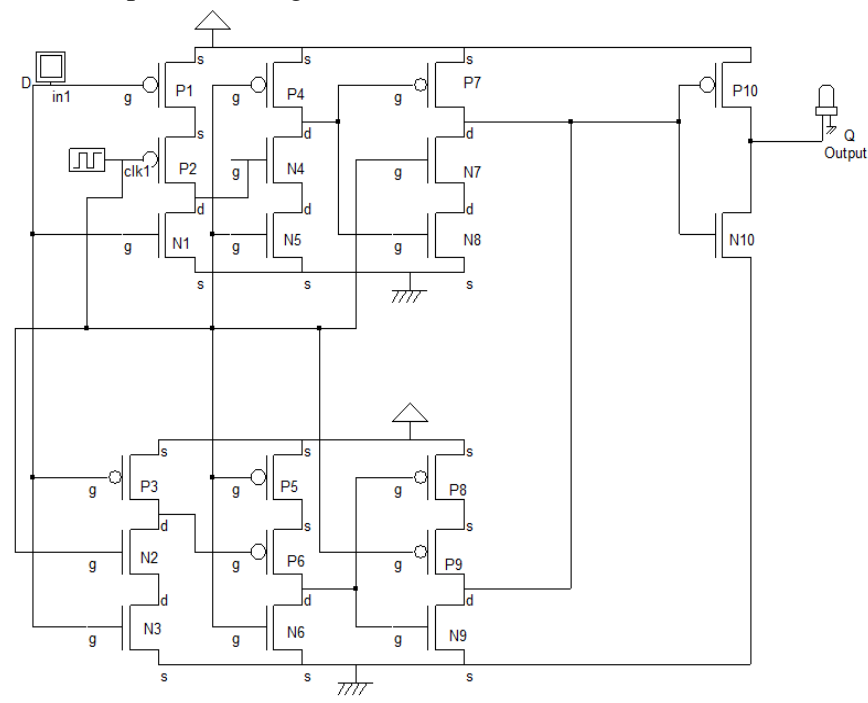

Fig.2 Conventional DE-TSPC D flip-flop.

The original circuit is depicted in Fig.2 but an extra inverter is added at the output to obtain a true logic and not the complemented logic. The true single phase clock dynamic CMOS circuit uses only one clock signal for synchronization and it also reduces complexity. As the circuit uses only one clock signal so that it is suitable for both static and dynamic CMOS circuits. In TSPC flip flop design a single global clock signal needs to be generated and distributed in order to simplify the design. The clocking system in the design may have single phase clock or multiple phase clocks. The clock phase controls the transfer of data between clock storage devices used in the synchronous VLSI digital systems [1011]. In the Fig. 2 output is same as input at both the rising and falling edge of the clock signal.

Now consider proposed design of DE-TSPC D flip-flop with 6T. Its design concept is based on positive edge triggered design of TSPC flip-flop as described in [10]. This design reduces the number of transistors in conventional design. So it reduces the area and power dissipation. Hence this design improves the performance. In this improved design 3PMOS and 3 NMOS transistors are used. Input is given at node D and output is computed at node Q. In this design single phase clock is given at the parallel attached PMOS and NMOS transistors. Schematic of proposed design of DE-TSPC D flipflop is presented in Fig.3. This double edge triggered TSPC flip-flop with 6 transistors working is explained in following two cases. Case I: when clock is low means $C L K=0$, (a) for input $(\mathrm{D})=1$, output $(\mathrm{Q})=1$; (b) for input (D) $=0$, output $(\mathrm{Q})$ $=0$. Case II: when clock is high means $\mathrm{CLK}=1$, (a) for input (D) $=1$, output $(\mathrm{Q})=1$; (b) for input (D) $=0$, output $(\mathrm{Q})=0$.

Fig.4 verifies the logic behaviour of the Double edge triggered TSPC flip-flop with 6 transistors circuit at $0.12 \mu \mathrm{m}$ technolgy. and presents logic simulation on DSCH which presents default gate and wire dealy is $0.030 \mathrm{~ns}$ and $0.070 \mathrm{~ns}$ respectivily. Elementary gate current is $500 \mathrm{~mA}$ with supply voltage of $1.2 \mathrm{~V}$.

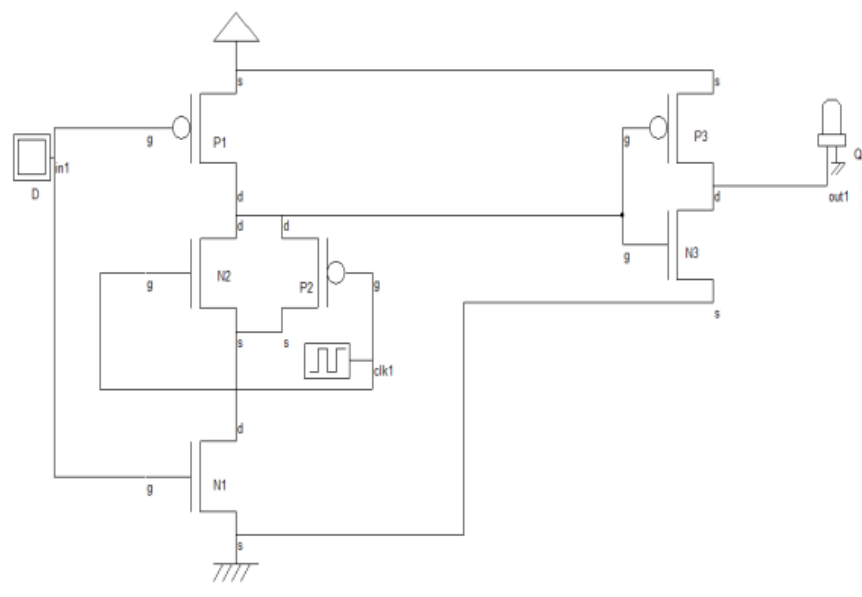

Fig. 3 Proposed DE- TSPC flip-flop with 6 T.

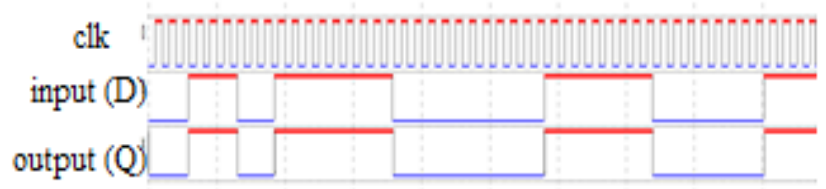

Fig.4 Timing simulation of proposed 6T DE-TSPC FF.

True single phase clocking D flip-flop has several advantages as it eliminates clock skew caused by different clock phases and clock signals are generated off-chip which significantly saves chip area and power consumption. But large portion of power is spent in pre-charging the internal nodes, which makes TSPC dynamic circuits less power efficient.

\section{MULTI THRESHOLD CMOS}

Multi-Threshold CMOS (MTCMOS) techniques is a highly accepted circuit technique that provide a solution to face the increase of static power dissipation. Multiple Threshold Complementary Metal Oxide Semiconductor (MTCMOS) technique enables the manufacturing of transistors with different threshold voltages $\left(\mathrm{V}_{\mathrm{th}}\right)$ on a single chip. A transistor with a lower $\mathrm{V}_{\mathrm{th}}$ offers higher switching speed but the penalty is given in the form of higher leakage current. Therefore, lower $\mathrm{V}_{\mathrm{th}}$ transistors or cells are used in critical delay paths of 
the design where higher speed is demanded [12-13]. The low $\mathrm{V}_{\text {th }}$ cells increase the leakage current even when circuit is dynamically inactive. A high $\mathrm{V}_{\text {th }}$ transistor known as "sleep transistor" can be added in series with the power supply network to further reduce leakage current during sleep mode. A common implementation of MTCMOS is to make use of sleep transistors; logic is supplied by virtual power rails as shown in Fig.5.

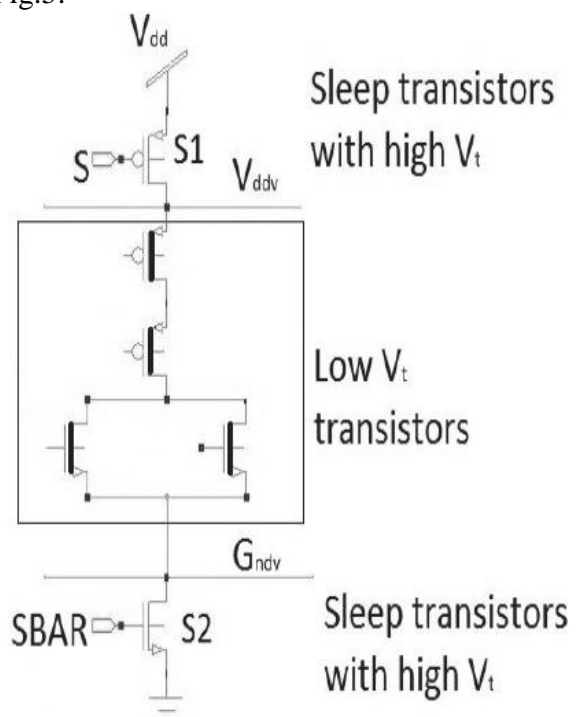

Fig.5 Power gating technique using Original MTCMOS

The other implementation is presented in Fig. 6. In this figure VDDV represent virtual power supply and VSSV represent virtual ground. And the circuitry between these virtual supplies is of low threshold voltage and the transistors between power supply and virtual power supply is of high threshold voltage. And transistor between virtual ground and ground is also of high thresholds voltage.

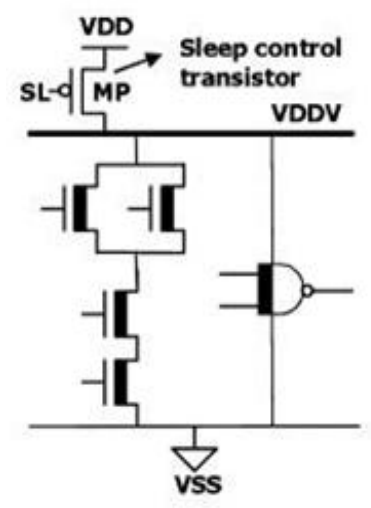

(a)

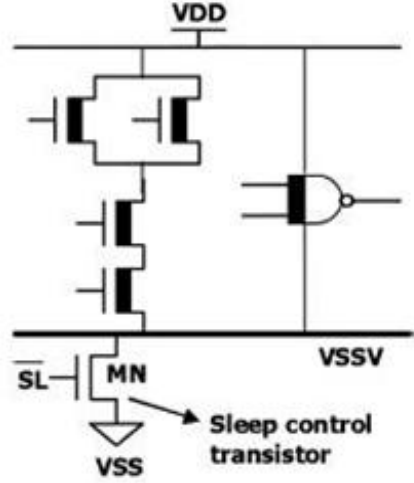

(b)
Fig.6 Various Implementation of MTCMOS (a) PMOS insertion MTCMOS (b) NMOS insertion MTCMOS

Multi threshold technique is used in the designing of Double edge triggered true single phase clocking D flip-flop for leakage minimization. This leakage current is the major drawback of these dynamic flip-flops.

\section{PROPOSED SCHEMATIC DESIGN}

The proposed schematic design of DE-TSPC D flip-flop is based on MTCMOS technique. The basic concept in the designing is using transistors with different threshold voltages
$\left(\mathrm{V}_{\mathrm{th}}\right)$. So designed double edge triggered flip-flop is having sleep transistors with high threshold voltage and rest of the transistors in the design is having low threshold voltage. The designed DE-TSPC flip-flop using original MTCMOS technique is shown in Fig.7.

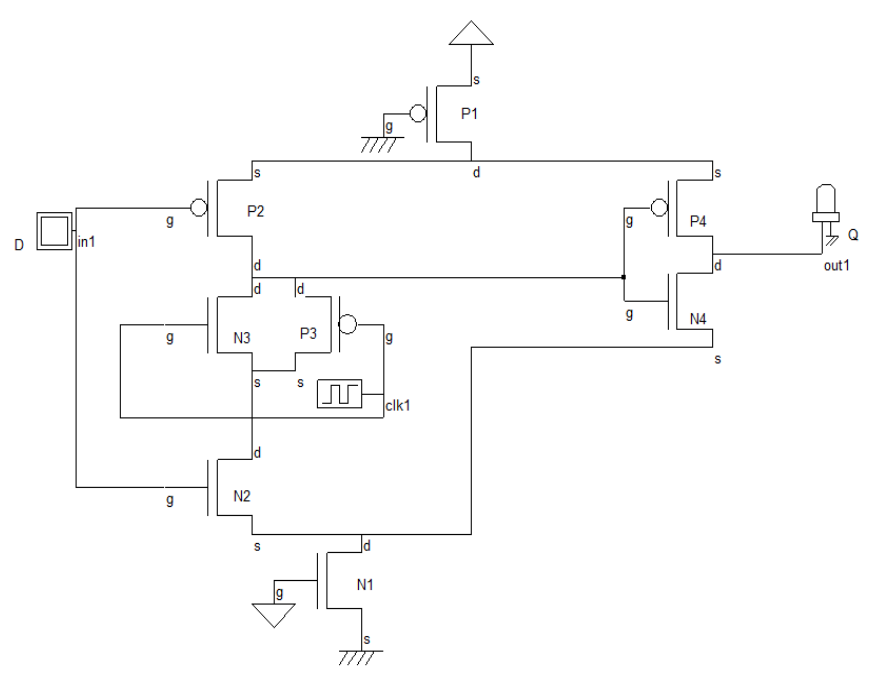

Fig.7 Design of proposed DE-TSPC FF using Original MTCMOS

In the above figure P1 and N1 transistor is having high threshold voltage known as sleep and sleep bar transistors respectively. These high threshold voltages transistors are useful on non critical delay path and also reduce leakage power without incurring any delay penalty. Now consider different cases for understanding the working: Case I: when the clock (clk) $=1$, input $\mathrm{D}=1$ and results in output $\mathrm{Q}=1$. Case II: when the clock (clk) $=1$, input $\mathrm{D}=0$ and it results in output $\mathrm{Q}=0$. Case III: when the clock (clk) $=0$, input $\mathrm{D}=1$ and it results in output $Q=1$. Case IV: when the clock $(\mathrm{clk})=1$, input $\mathrm{D}=0$ and it results in output $\mathrm{Q}=0$.

Fig.8 verifies the logic behaviour of the Double edge triggered TSPC flip-flop using MTCMOS technique at $0.12 \mu \mathrm{m}$ technolgy.

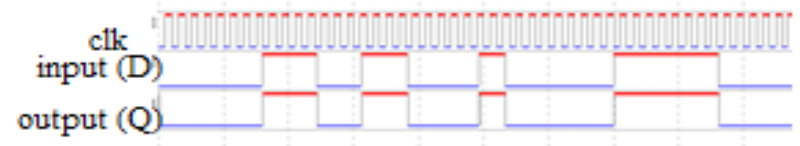

Fig.8 Timing simulation of proposed DE-TSPC FF using original MTCMOS

Now consider another implementation which is based on insertion of NMOS in MTCMOS. In this implementation for virtual power supply a voltage source of $1.2 \mathrm{~V}$ is used as shown in Fig.9. 


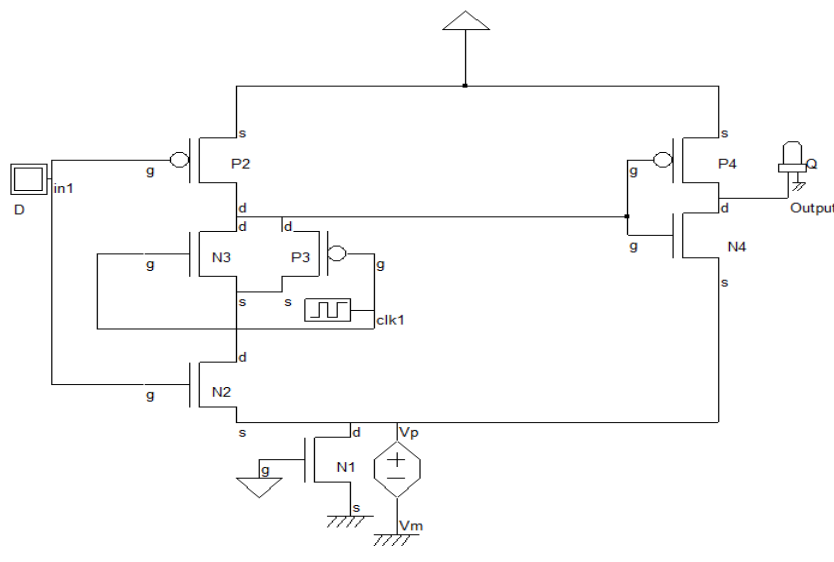

Fig.9 Proposed DE-TSPC FF using NMOS insertion MTCMOS

Fig.10 verifies the logic behaviour of the Double edge triggered TSPC flip-flop using MTCMOS technique at $0.12 \mu \mathrm{m}$ technolgy.

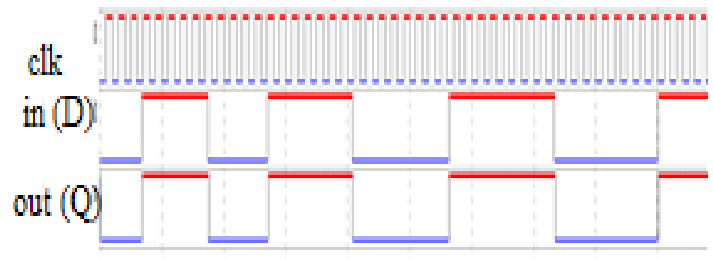

Fig.10 Timing waveform of proposed DE-TSPC FF with NMOS insertion MTCMOS

\section{LAYOUT SIMULATION}

The layout design rule describes how the small features can be and how closely they can be packed in particular manufacturing process. Different logical layers are used by the designers to generate the layout. Layout or mask design describes an integrated circuit in terms of planar geometric shapes which interprets the patterns of metal, oxide, or semiconductor layers that build up the components of the integrated circuit. Main job of a layout designer is to place and connect all the components to make a chip so that they meet all criteria of design. Main constraints of layout design rule are size, manufacturability and performance [14]. Physical layout of conventional double edge triggered TSPC D flip-flop is designed using MICROWIND 3.1 and simulation is performed using BSIM4 model. Schematic of conventional DE-TSPC flip-flop is designed using DSCH 3.1 tool. After compiling the verilog file, Layout of the circuit is achieved in the MICROWIND. A verilog file is a kind of netlist consisting all the components and connections used in designing of a circuit. Layout of conventional double edge triggered TSPC flip-flop is shown in Fig.11.

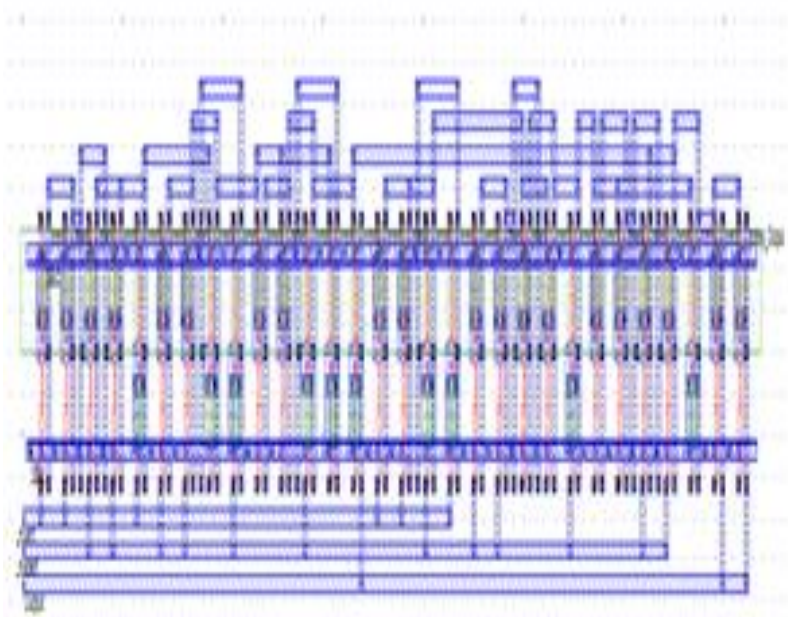

Fig.11 Layout of conventional DE-TSPC D flip-flop

Analog simulation waveform of conventional DE-TSPC D flip-flop is shown in Fig.12. These waveforms are obtained after simulating the waveform using BSIM4 model. This analog simulation presents power dissipation and delay. And the simulation presents that conventional DE-TSPC D flipflop has power dissipation of $28.87 \mu \mathrm{W}$ at $1.2 \mathrm{~V}$.

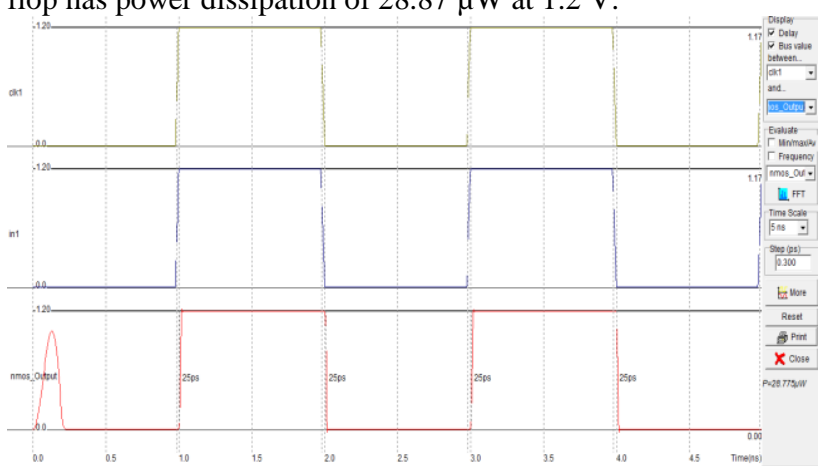

Fig.12 Layout simulation of conventional DE-TSPC FF

Layout structure of DE-TSPC D flip-flop with fewer transistors is obtained after compiling the verilog. Different metal layers are used, which includes metal1, metal 2 up to six. For gate poly-silicon layer is used. $\mathrm{V}_{\mathrm{DD}}$ and ground are made using metal1. Layout cell design is shown in Fig.13.

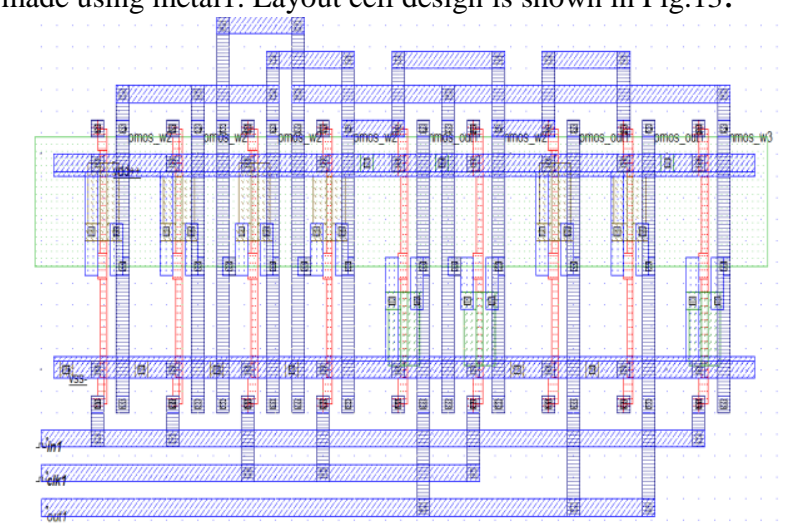

Fig.13 Layout of proposed 6T DE-TSPC D FF

Layout simulation of proposed DE-TSPC D flip-flop with fewer Transistors circuit is shown in Fig. 14. The waveforms verify the correct logic of the circuit. Range of the voltage used for analog signal is from $0 \mathrm{~V}$ to $1.2 \mathrm{~V}$. These waveforms 
provide significant information, which includes power, delay, frequency and maximum drain current. Thus power dissipation is $24.585 \mu \mathrm{W}$ at $1.2 \mathrm{~V}$ as shown in following figure.

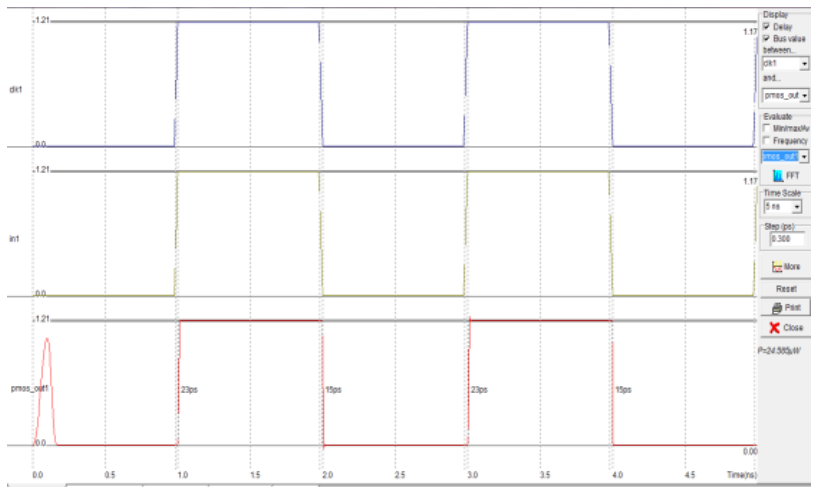

Fig.14 Layout simulation of 6T DE-TSPC D flip-flop

Layout design of proposed DE-TSPC D flip-flop using original MTCMOS means it make use of sleep and sleep bar transistors of high threshold voltage is shown in Fig. 15. Regular layout style is used in order to simplify the overall geometry and the signal routing. Layout is drawn at $0.12 \mu \mathrm{m}$ technology and at this foundry the value of lambda is $0.06 \mu \mathrm{m}$. For input poly to metall and metal 1 and metal 2 contact is used. For output metall to metal 2 contact is used.

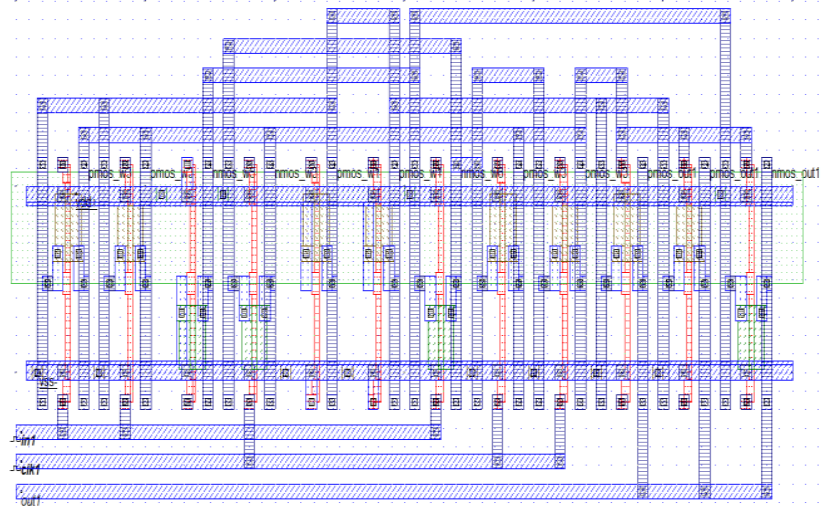

Fig.15 Layout of proposed DE-TSPC D FF using original MTCMOS

Analog simulation is performed on the layout of proposed DE-TSPC D flip-flop using sleep transistors. Fig.16 shows time domain simulation of circuitry in which Logic ' 0 ' corresponds to a zero voltage and logic ' 1 ' corresponds to $1.2 \mathrm{~V}$. Simple clocks are applied as inputs and select lines. This circuitry has power dissipation of $12.235 \mu \mathrm{W}$ at 1.2 . V.

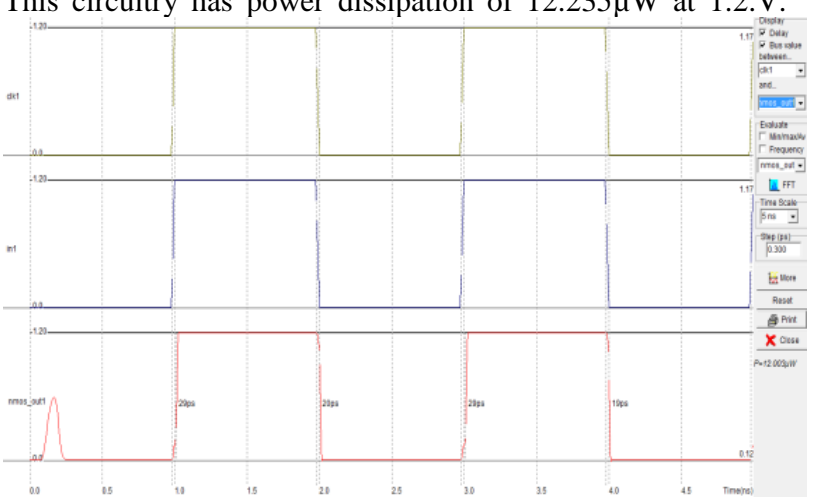

Fig.16 Layout simulation of proposed DE-TSPC D FF using original MTCMOS
Layout design of DE-TSPC D flip-flop using MTCMOS Technique in which a voltage source is used in parallel with NMOS transistor is shown in Fig.17. Layout is drawn at $0.12 \mu \mathrm{m}$ technology.

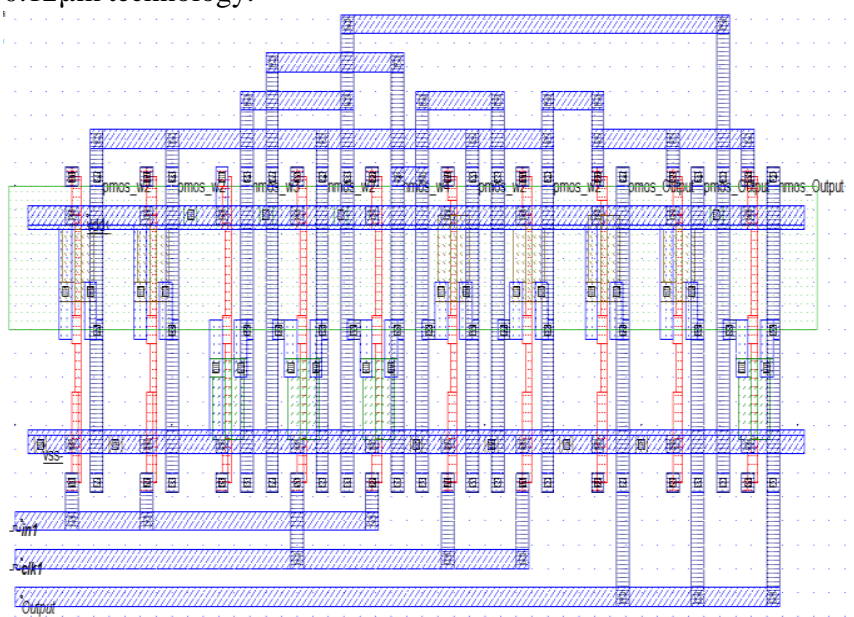

Fig.17 Layout simulation of proposed DE-TSPC D FF using NMOS insertion in MTCMOS

Analog simulation is performed on the layout of DE-TSPC D Flip-Flop using MTCMOS technique in which a voltage source is used in parallel with NMOS transistor. Fig. 18 shows time domain simulation of circuitry. This circuitry has power dissipation of $11.845 \mu \mathrm{W}$ at $1.2 . \mathrm{V}$ as shown in following figure.

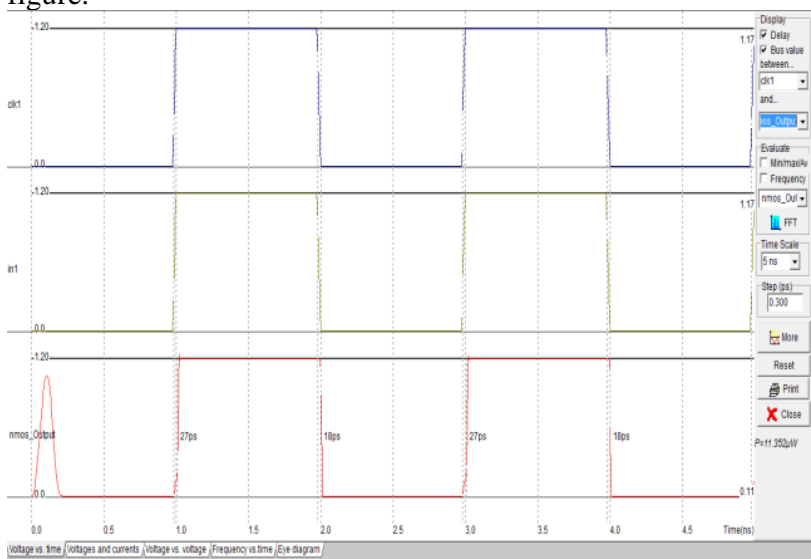

Fig. 18 Layout simulation of NMOS Insertion MTCMOS

\section{RESULTS \& DISCUSSION}

This presents an extensive comparison of different designs of dynamic TSPC D flip-flops. These dynamic flip-flops are more sensitive to noise and leakage current so various design are proposed in order to reduce lekage power by using MTCMOS technique. All these designs are designed using DSCH and simulation is performed on Microwind 3.1 tool with $120 \mathrm{~nm}$ technology.

Consider the static power dissipation of proposed DETSPC D flip-flop with MTCMOS and without MTCMOS technique. In Table 1 DE-TSPC flip-flop with and without MTCMOS technique are compared at different supply voltage with a constant temperature of $27^{\circ} \mathrm{C}$. Table 1 shows that MTCMOS technique reduces the problem of leakage as it minimizes static power dissipation in the of DE-TSPC D flipflop. 
Table 1 Comparison of Proposed DE-TSPC FF with and without MTCMOS technique

\begin{tabular}{|c|c|c|c|}
\hline Parameter & \multicolumn{3}{|c|}{ Power Dissipation $(\mu \mathrm{W})$} \\
\hline $\begin{array}{c}\text { Supply } \\
\text { Voltage }\end{array}$ & $\begin{array}{c}\text { Proposed 6T } \\
\text { DE-TSPC } \\
\text { without } \\
\text { MTCMOS }\end{array}$ & $\begin{array}{c}\text { Proposed } \\
\text { DE-TSPC } \\
\text { using } \\
\text { original } \\
\text { MTCMOS }\end{array}$ & $\begin{array}{c}\text { Proposed } \\
\text { DE-TSPC } \\
\text { using } \\
\text { NMOS } \\
\text { insertion in } \\
\text { MTCMOS }\end{array}$ \\
\hline $1 \mathrm{~V}$ & 11.860 & 9.927 & 9.442 \\
\hline $1.2 \mathrm{~V}$ & 24.585 & 12.235 & 11.845 \\
\hline $1.4 \mathrm{~V}$ & 31.122 & 29.832 & 29.437 \\
\hline $1.6 \mathrm{~V}$ & 109.00 & 35.206 & 35.724 \\
\hline $1.8 \mathrm{~V}$ & 565.00 & 44.468 & 39.680 \\
\hline $2 \mathrm{~V}$ & 1258 & 95.573 & 56.193 \\
\hline
\end{tabular}

Fig.19 presents the variation of power dissipation with respect to supply voltage for proposed DE-TSPC flip-flop with and without MTCMOS technique and it also verifies the results of Table 2 .

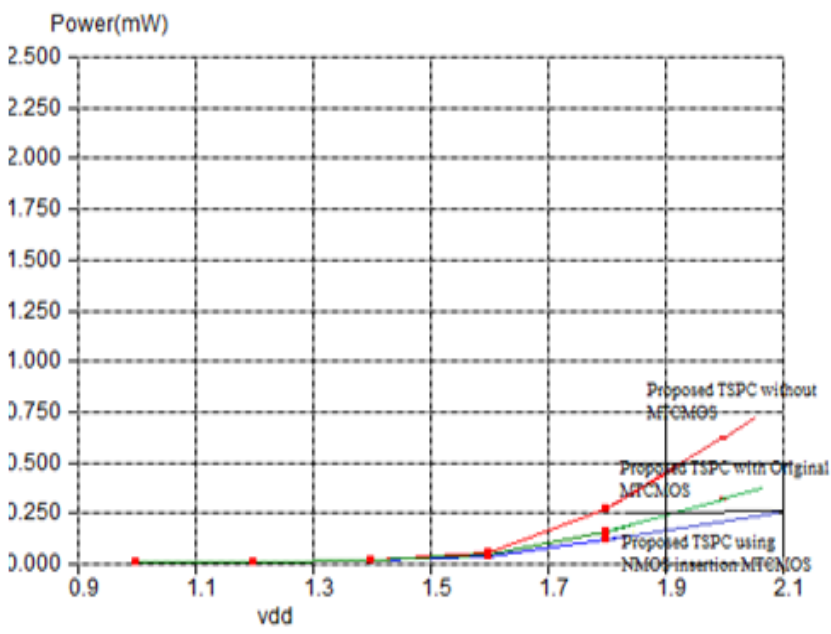

Fig.19 Power Consumption vs Supply Voltage for proposed DE-TSPC D flip-flop

Static power dissipation of dynamic TSPC D flip-flop with eighteen transistors i.e. the conventional design proposed by Wang [9] and DE-TSPC D flip-flop with six transistors is presented in Table 2. Static power dissipation of conventional DE-TSPC D flip-flop is higher than 6T DE-TSPC D flipflop.This table presents an extensive comparison of positive edge triggered and double edge triggered TSPC flip-flops.

Table 2 also presents the comparison of the positive edge triggered 11 transistors TSPC [7] and 5 transistors TSPC D flip-flop [6]. This comparison is performed at different supply voltage with a room temperature of $27^{0} \mathrm{C}$. As the supply voltage increases power consumption increases in the same proportion. Hence positive edge triggered 11T TSPC D flipflop design has high power dissipation.
Table 2 Static power dissipation for different TSPC D FF

\begin{tabular}{|c|c|c|c|c|c|c|}
\hline \multirow{2}{*}{ Parameter } & \multicolumn{5}{|c|}{ Power dissipation $(\mu \mathrm{W})$} \\
\hline $\begin{array}{c}\text { Supply } \\
\text { Voltage }\end{array}$ & $\begin{array}{c}11 \mathrm{~T} \text { PE } \\
\text { TSPC } \\
\text { FF [7] }\end{array}$ & $\begin{array}{c}\text { 5T PE } \\
\text { TSPC } \\
\text { FF [6] }\end{array}$ & $\begin{array}{c}18 \mathrm{~T} \\
\text { DE } \\
\text { TSPC } \\
\text { FF [9] }\end{array}$ & $\begin{array}{c}\text { 6T } \\
\text { FF }\end{array}$ & $\begin{array}{c}\text { Using } \\
\text { Original } \\
\text { MT- } \\
\text { CMOS }\end{array}$ & $\begin{array}{c}\text { NMOS } \\
\text { insertion } \\
\text { MT- } \\
\text { CMOS }\end{array}$ \\
\hline $1.2 \mathrm{~V}$ & 519.0 & 25.2 & 28.8 & 24.585 & 12.235 & 11.845 \\
\hline $1.4 \mathrm{~V}$ & 1448 & 34.1 & 75.7 & 31.122 & 29.832 & 29.437 \\
\hline $1.6 \mathrm{~V}$ & 1936 & 102.9 & 232.0 & 109.00 & 35.206 & 35.724 \\
\hline $1.8 \mathrm{~V}$ & 3081 & 655 & 925 & 565.00 & 44.468 & 39.680 \\
\hline $2.0 \mathrm{~V}$ & 4777 & 1246 & 1958 & 1258 & 95.573 & 56.193 \\
\hline
\end{tabular}

Fig.20 and Fig. 21 verifies the results of Table 2. It presents impact of supply voltage variation on power dissipation for different logic style of dynamic TSPC flip-flop.

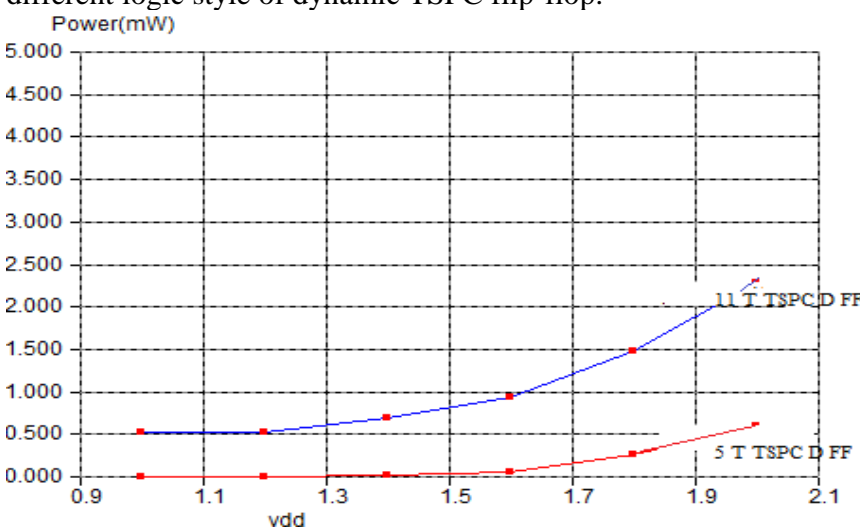

Fig.20 Power Consumption vs Supply Voltage for PETSPC D flip-flop

In Fig. 20, RED line presents variation of power consumption with respect to voltage for $5 \mathrm{~T}$ design of positive edge triggered TSPC D flip- flop; BLUE line presents variation of power consumption with respect to voltage for $11 \mathrm{~T}$ design of positive edge triggered TSPC D flip-flop.

In Fig. 21, BLUE line in the graph presents variation of power consumption with respect to voltage for conventional design of double edge triggered TSPC D flip- flop; RED line presents variation of power consumption with respect to voltage for 6T design of double edge triggered TSPC D flip-flop. Hence the 6T design of DE-TSPC flip-flop indicates low power dissipation. 


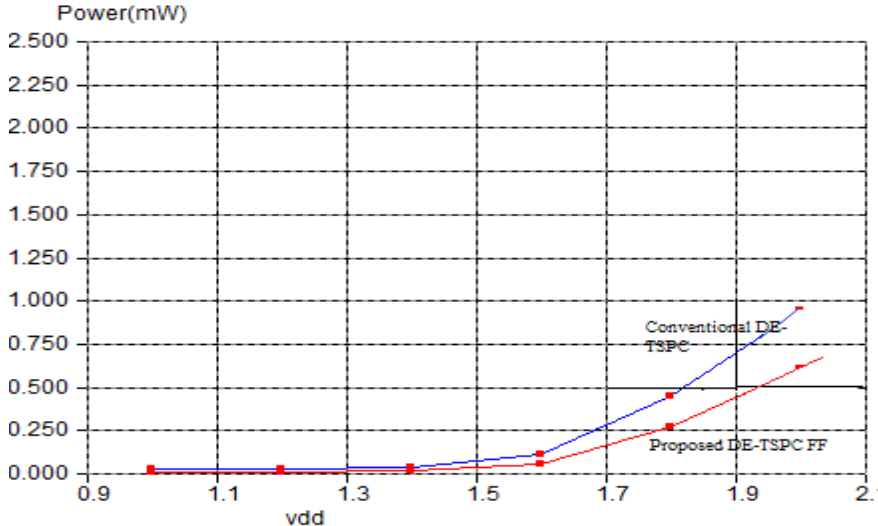

Fig.21 Power Consumption vs Supply Voltage for DETSPC D flip-flop

Fig. 22 shows the variation of maximum drain current with supply volatge for double edge triggered TSPC D flip-flop using MTCMOS technique. Drain current increases linearly with supply voltage. It has been observed that drain current is minimum for design of origional MTCMOS. I mA

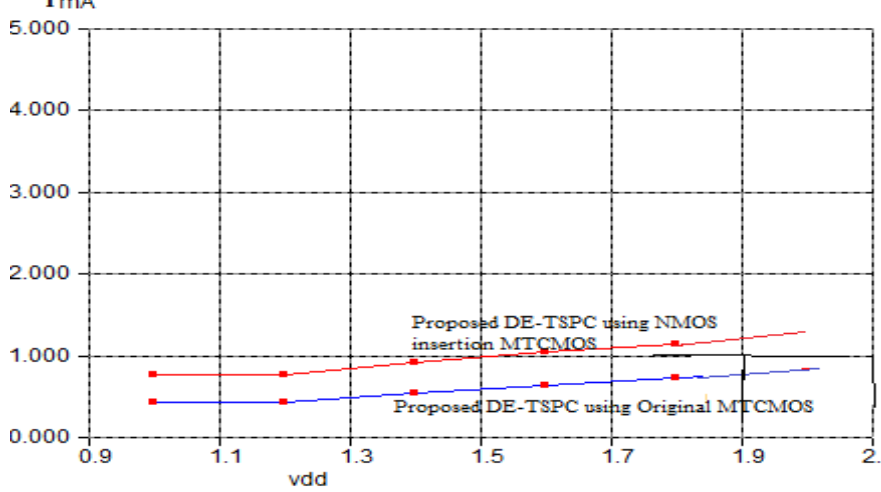

Fig.22 Maximum drain current vs supply voltage graph for MTCMOS design of Proposed TSPC D flip-flop

Fig. 23 shows the variation of maximum drain current with supply volatge for double edge triggered TSPC D flip-flop with $18 \mathrm{~T}$ and 6T. Drain current increases linearly with supply voltage [15]. It has been observed that drain current is minimum for 6T design of TSPC D flip-flop as shown in figure with RED line.

$$
\operatorname{ImA}
$$

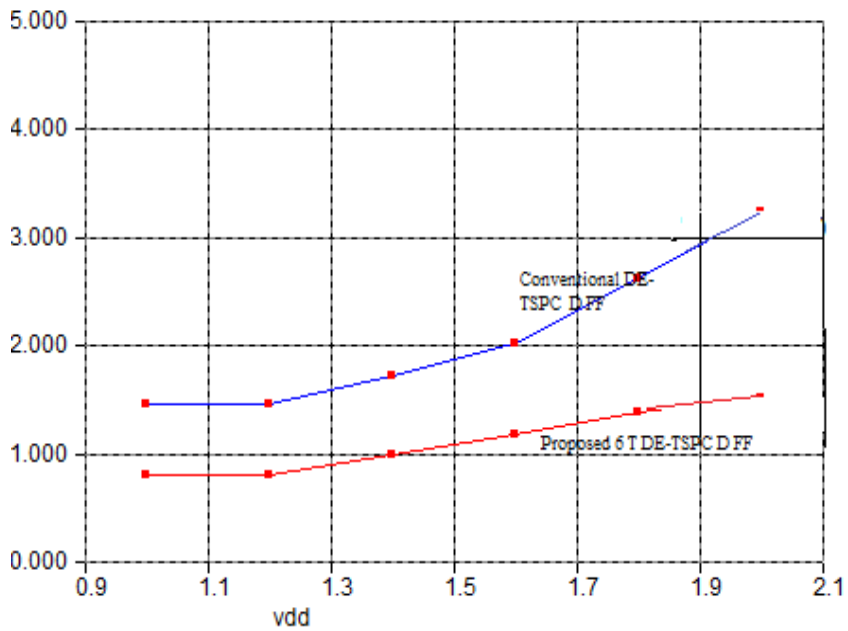

Fig.23 Maximum drain current vs supply voltage graph for DE-TSPC flip-flops
Fig. 24 presents variation of maximum drain current with supply volatge for various double edge triggered TSPC D flipflops with and without MTCMOS technique. Thus MTCMOS designs shows good results as it minimizes the leakage current.

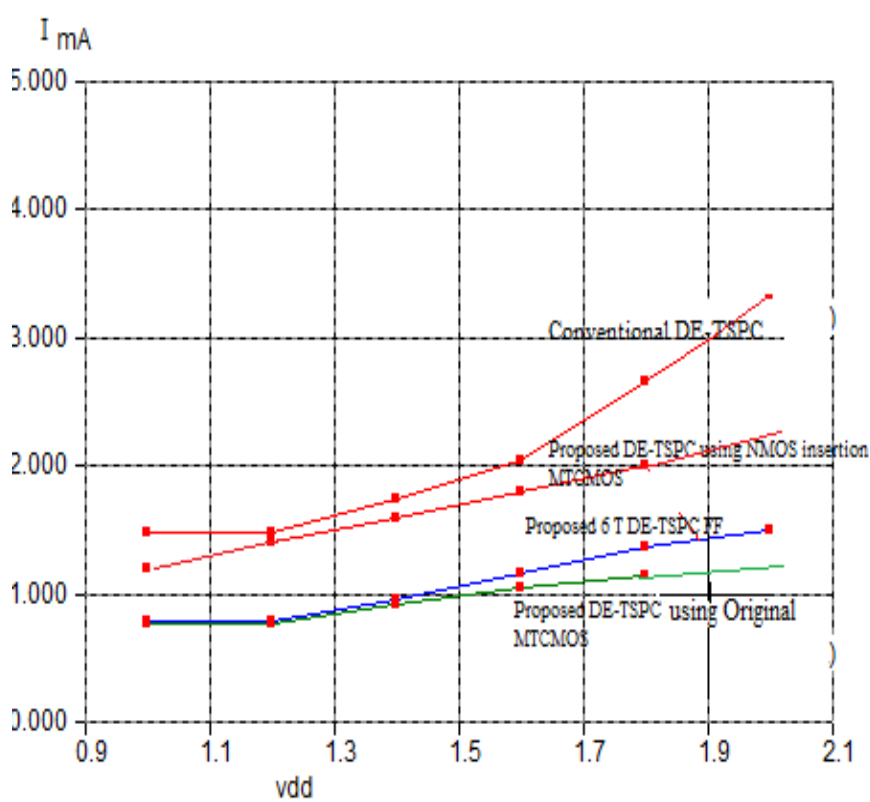

Fig.24 Maximum drain current vs supply voltage comparison

In the Table 3 figure of merit (FOM) is defined by power delay product means the circuit having least power delay product has highest FOM and will give best performance.

Table.3 Drain current \& FOM comparison at 1.2V

\begin{tabular}{|c|c|c|c|c|c|c|}
\hline \multirow[t]{2}{*}{ Parameter } & \multirow{2}{*}{$\begin{array}{l}11 \mathrm{~T} \\
\text { PE } \\
\text { TSPC } \\
\text { FF [7] }\end{array}$} & \multirow{2}{*}{$\begin{array}{c}5 \mathrm{~T} \text { PE } \\
\text { TSPC } \\
\text { FF [6] }\end{array}$} & \multirow{2}{*}{\begin{tabular}{|c}
$18 \mathrm{~T}$ \\
DE \\
TSPC \\
FF [9]
\end{tabular}} & \multicolumn{3}{|c|}{ Proposed DE-TSPC } \\
\hline & & & & $\begin{array}{l}6 \mathrm{~T} \\
\mathrm{FF}\end{array}$ & $\begin{array}{c}\text { Using } \\
\text { Origina } \\
\text { MT- } \\
\text { CMOS }\end{array}$ & $\begin{array}{c}\text { NMOS } \\
\text { insertion } \\
\text { MT- } \\
\text { CMOS }\end{array}$ \\
\hline $\begin{array}{l}\text { Max. } \\
\text { Drain } \\
\text { Currenet } \\
(\mathrm{mA})\end{array}$ & 3.090 & 1.222 & 1.481 & 0.812 & 0.443 & 0.584 \\
\hline $\begin{array}{l}\text { FOM } \\
\text { (fJ) }\end{array}$ & 8.823 & 0.3804 & 0.7194 & 0.590 & 0.3720 & 0.3292 \\
\hline
\end{tabular}

So positive edge triggered TSPC D flip-flop with 5T are better than $11 \mathrm{~T}$ as it has least power delay product. Similarly 6T DE-TSPC D flip-flop are better in performance than conventional DE-TSPC D flip-flop. Now cosider MTCMOS designing of DE-TSPC D flip-flop, NMOS insertion MTCMOS design of TSPC D flip-lop are better in performance than the original MTCMOS design of TSPC D flip-flop. it has been observed that DE-TSPC flip-flops using MTCMOS technique are best in performance as they have highest figure of merit (FOM).

Graph in Fig. 25 shows variation in power dissipation of different double edge triggered TSPC D flip-flop. this graph reveals that power dissipation for conventional DE-TSPC D flip-flop is very high among all. All the design technologies are already discussed in above sections. 


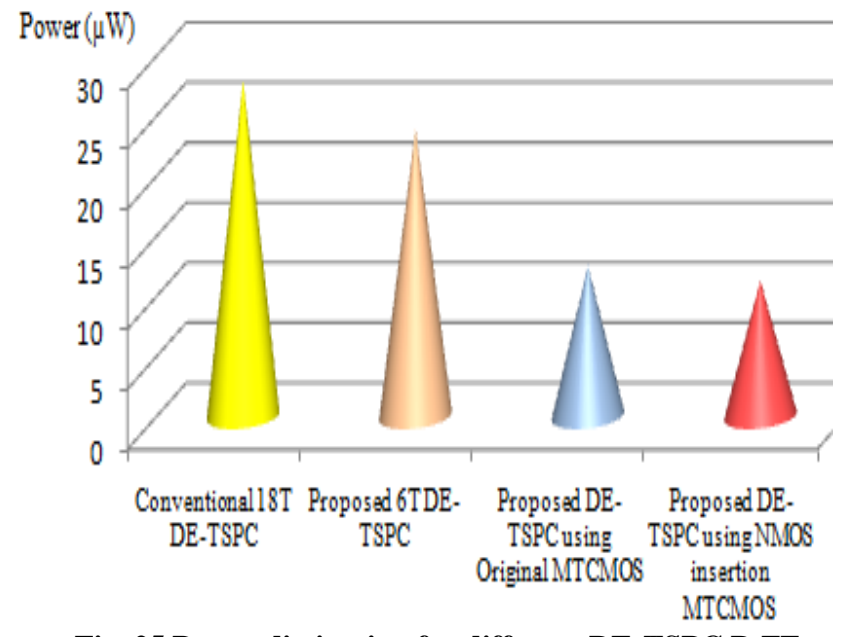

Fig. 25 Power dissipation for different DE-TSPC D FF

\section{CONCLUSION}

The true single-phase clock (TSPC) type is a common dynamic flip-flop variety which performs the flip-flop operation with low power and at high speeds. However, dynamic flip-flops will typically not work at low clock speeds, required enough time, leakage paths may discharge the parasitic capacitance enough to cause the flip-flop to enter invalid states. An efficient design of double edge triggered True Single Phase Clocking (TSPC) D flip-flop is presented. The main advantage of TSPC dynamic CMOS circuit is that it is small in area, no clock skew and can achieve the higher clock rates. Hence it is a promising storage element in low power VLSI designs. The originally developed TSPC flip-flop are very sensitive to the clock slope and large portion of power is spent in pre-charging the internal nodes, which makes TSPC dynamic circuits less power efficient. In the conventional CMOS design, high leakage current is becoming a significant contributor to power dissipation. To overcome the existing problem of CMOS TSPC D flip-flop, a Multithreshold CMOS (MTCMOS) technology is used for leakage minimization. The proposed schematic design of DE-TSPC D flip-flop is based on MTCMOS technique. The basic concept in the designing is using transistors with different threshold voltages $\left(\mathrm{V}_{\mathrm{th}}\right)$. The MTCMOS design of TSPC flip -flop is having least power delay product (PDP) thus highest figure of merit hence it gives best performance and it can be used in several applications like level converters, microprocessors, clocking system counter etc. It is recorded that proposed original MTCMOS implmentation and NMOS insertion in MTCMOS design of TSPC D flip-flop saves static power $57.517 \%$ and $58.871 \%$ as compared to conventional DETSPC D flip-flop respectively at $1.2 \mathrm{~V}$. Further these designs are compared with 6T TSPC D flip-flop and improvement is obtained in terms power consumption as they saves $50.23 \%$ and $51.82 \%$ respectively at $1.2 \mathrm{~V}$. Proposed DE-TSPC flopflop shows good performance with supply voltage vs drain current variations as compare to conventional DE-TSPC flipflop.

\section{REFERENCE}

[1] S. Unger and C. Tang, "Clocking Schemes for Very High-Speed Digital Systems", IEEE Transaction on Computers, Vol.: C-35, pp. 880-895, October 1986.

[2] M. Pedram, "Power minimization in IC Design: Principles and applications," ACM Transactions on Design
Automation of Electronic Systems, Vol. 1, pp. 3-56, January 1996.

[3] B. Nikolic, "Design in The Power Limited Scaling Regime," IEEE Transaction on Electronic Devices, Vol. 55, No. 1, pp. 71-83, January 2008.

[4] Neil H. E. Weste, David Harris, Ayan Banerjee, CMOS VLSI DESIGN: A Circuits and Systems Prespective, Third Edition, pp. 129-131, 2009.

[5] S. Tahmasbi Oskuii, A. Alvandpour, "Comparative Study on Low-Power High- Performance Standard- Cell FlipFlops", Microelectronics: Design, Technology, and Packaging, in Proceedings of the International Society for Optics and Photonics (SPIE), Vol. 5274, pp. 390-398, 2004.

[6] Surya Naik and Rajeevan Chandel, "Design of a Low Power Flip-Flop Using CMOS Deep Submicron Technology", IEEE, International Conference on Recent Trends in Information, Telecommunication and Computing (ITC), pp. 253-256, 2010.

[7] Neil H. E. Weste, David Harris, Ayan Banerjee, a book on "CMOS VLSI Design a Circuits and Systems Perspective", Pearson Education, 3rd Edition, pp. 275275, 2009.

[8] J. A. Butts and G.S. Sohi, "A Static Power Model for Architects", IEEE, In Proceedings of the $33^{\text {rd }}$ Annual International Symposium on Micro-architecture, pp. 191201, 2000.

[9] J. S. Wang, "A New True-Single-Phase-Clocked Double Edge Triggered Flip-Flop for Low-Power VLSI Design", in Proceedings of IEEE International Symposium on Circuit and Systems, pp. 1896-1899, 12 June 1997.

[10] M Afghahi and C. Svenson, "A Unified Single Phase Clocking Scheme for VLSI Systems", IEEE Journal Solid-State and Circuits, Vol. 25, pp. 255-233, February 1990.

[11] B. Pontikakis and M. Nekhili. "A New Area Efficient Split- Output TSPC CMOS Latch for High Speed VLSI Applications", IEEE, International Conference of Microelectronics (ICM), pp. 101-104, 2002.

[12] L. Wei, Z. Chen, M. Johnson, K. Roy and V. De, "Design and Optimization of Low Voltage High Performance Dual Threshold CMOS Circuits," IEEE Transactions on Very Large Scale Integration (VLSI) Systems, pp. 16 - 24, March 1999.

[13] S. Mutoh et al., "A 1V Multi-Threshold Voltage CMOS DSP with an Efficient Power Management Technique for Mobile Phone Application”, IEEE Journal of Solid-State Circuits, Vol. 31, No.11, pp. 1795-1802, 1996.

[14] Etienne Sicard, Sonia Ben Dhia, Syed Mahfuzul Aziz, "Teaching CMOS Circuit Desig in Nano-scale Technologies Using MICROWIND", Proceedings of the European workshop on microelectronics education, pp. 78-83, May 2010.

[15] Jayanth Srinivasan, "An Overview of Static Power Dissipation", CiteSeer public search engine and digital libraries for scientific and academic papers in the fields of computer and information science, pp. 1-7, 30 August 2011. 